In: Kant and the Laws of Nature, eds. M. Massimi \& A. Breitenbach, Cambridge UP, 2016.

\title{
Metaphysical foundations of neoclassical mechanics
}

\begin{abstract}
MARIUS STAN
I examine here if Kant's metaphysics of matter can support any late-modern versions of classical mechanics. I argue that in principle it can, by two different routes. I assess the interpretive costs of each approach, and recommend the most promising strategy, viz. the mass-point approach.
\end{abstract}

The most influential current accounts of Kant's philosophy of physics have it that our latter-day science of nature has shown his doctrine to be too narrow, thus no longer tenable in its original strong form. Its most glaring limitation is the geometric and chronometric structure-or determinate space and time content - to which Kant wedded it. Modern gravitation theory has shown that structure to be valid just in the classical limit. Thus, prominent interpreters have concluded, Kant's philosophical foundation must be read in light of the best science of his time, not ours-specifically, in dialogue with Newton's Principia, the high noon of early modern physics.

Suppose we are in the 'classical belt' of the world, where masses and speeds are low enough that relativistic theory is not needed. Has Kant's philosophy been refuted for that realm too? I claim that it has not. His views, I argue, remain as relevant as classical mechanics is alive, despite some premature reports of its demise in the wake of Einstein. To see my point, however, we must turn away from space- or time structure and the Transcendental Aesthetic that legitimizes it. The most viable part of Kant's foundations, I suggest, is the transcendental logic -in its more determinate form as a constitution theory for objecthood in mechanics. ${ }^{1}$

\footnotetext{
${ }^{1}$ Constitution theory is Kant's account of the 'forms' that constitute the object of experiencespecifically, the two forms of sensibility, and the conceptual structure supplied by the understanding. Here I am interested in the latter. I refer to Kant's Metaphysical Foundations of Natural Science simply as 'Foundations,' and cite it by volume and page number in the Academy Edition, from Kant 2004.
} 
Is that not a tall order? Will it not require me to try and defend anew the completeness of Kant's table of categories? No, not really. I am not asking here, what in the Transcendental Analytic can still explain the possibility of experience. Rather, I follow the Marburg neo-Kantians as I carve a new path alongside theirs. From Hermann Cohen to Michael Friedman, they have investigated how radical change of inertial structure affects Kantian doctrine. I bracket their question entirely as I pursue my novel path. That is, I take classical structure-Galilean kinematics, Euclidean parallel transport, and absolute simultaneity - for granted, and focus instead on the axiomatic structure of mechanical theory. More exactly, I ask: what is the broadest genus of object for modern classical mechanics that we can anchor in Kant's categories and sensible forms? This question, I claim, has a cogent answer that shows Kant's metaphysics of material nature is viable and relevant, if we restrict it to its proper domain.

From my new vantage point, there is another ground for optimism. The conceptual basis of modern mechanics is an edifice with four parts: 1) fundamental kinematics, 2) a matter theory, 3) dynamical laws and 4) a doctrine of objectivity. Kant had already anticipated this architecture, and called these parts Phoronomy, Dynamics, Mechanics, and Phenomenology, respectively. If we look at them from the perspective I have outlined, new insight emerges into his constitution theory of mechanical objects-and a new answer becomes possible to the question: Do Kant's metaphysical foundations survive the challenge of modern science?

The question also bears directly on the central theme in this volume. We may regard Kant's constitutive principles in Foundations as laws broadly conceived: basic kinematic laws, laws of material constitution, general dynamical laws, and laws of mechanical objectivity. Then we can rephrase my question above as follows: are the laws of his 'metaphysics of corporeal nature' still the laws of nature in a classical regime? Answer: yes.

But, I must qualify my subject. Mechanics post-Principia is really the confluence of two broad streams of theory, viz. Newton-Euler dynamics and 'analytic' mechanics. I set the latter entirely aside here. It poses serious challenges to my project, far from easy to solve, though I hope not intractable. I 
acknowledge them, and move on to examine Kant's chances of grounding the former. That holds out more promise and reward.

Still, the path to my conclusion is not apparent. Even circumscribed as Newton-Euler theory, modern mechanics rests on two distinct fundamental objects, viz. the mass-point and the deformable continuum. ${ }^{2}$ Each object by itself supports a comprehensive theory of classical mechanics. And, neither is explainable in terms of the other-they are mutually irreducible objects. Thus, from the stance I take here, we cannot answer my question above directly. So, I respond with a blend of reconstruction and hermeneutics. I spell out modern constitutive assumptions for the two objects above, and examine two respective strategies for embedding them in Foundations. Against that backdrop, I recommend the least disruptive path for the interpreter. Ipso facto that is also a case for the viability of Kant's metaphysical foundations.

Fortunately, Kant himself will help me in my enterprise. It is often thought that Foundations is a univocal account of a single kind of mechanical object. As I will show, that is not true. Kant speaks there in two voices, perhaps unwittingly, and he wavers between two species of object, discrete and continuous. This raises the question, which is really his considered view-or, at least, which view is easier to defend on his behalf? My study thus is not just a case for Kant's continued relevance; it is also a fresh look at the deep structure of Foundations, which Kant obscures with his architectonic-driven mode of presentation.

However, his vacillation is hard to see without help from modern theory. Accordingly, I start with it, by spelling out material constitution, fundamental kinematics, dynamical laws, and objectivity criteria for a mass-point, the first kind of mechanical object ( $§ 1$ ). And, I do the same for the second kind, viz. the deformable continuum ( $(2)$. Then I turn to Kant, and examine the resources for grounding either object in his constitution theory $(\S 3)$. I end by assessing and recommending the most promising approach $(\S 4){ }^{3}$

\footnotetext{
${ }^{2}$ There is a third unit of matter, the rigid body, which I leave out here. Kant argued resolutely against it, and it would be hard to brush that aside. And, the rigid body is the preferred ontology of analytic mechanics, which I have set aside for now, as I explained above.

${ }^{3}$ Sections 1 and 2 are synoptic capsules that rely variously on Hamel 1909, Joos 1934, Malvern 1969, Truesdell 1991, Gurtin, Fried \& Anand 2010, and Wilson 2013.
} 
'Neoclassical' denotes a certain presentation of mechanics, more rigorous than the partial and often implicit 'classical' version of Newton and Kant, but less formal than the 'post-classical' axiomatizations of Noll and his school.

\section{The mass-point approach}

Kant's Dynamics is really a matter theory, and so its modern equivalent would be an account of material constitution. (I mean that as in physical theory, not intuition-based analytic metaphysics.) In current terms, one type of basic object, or unit of matter, is an entity the size of a point. This zero-sized object is endowed with two causally efficient, scalar-valued properties: inertial mass and charge (of various types). Mass grounds impenetrability in that no two masses can be superimposed, or made to occupy the same point-sized place simultaneously; resistance to acceleration by another mass-point; and the ability to carry and transfer momentum. Charges give rise to scalar fields around the point, and they in turn induce fields of acceleration at every location for which they are defined. Some theorists capture these facts by saying that a point mass has no true size, just an effective volume. On this view, macroscopic bodies are discrete ensembles, and mass-points are their ultimate actual parts.

Kinematics. An unconstrained mass-point has three degrees of freedom: three independent parameters specify its position completely. A motion is a change in any degree of freedom; there is no intrinsic spin.

Because point masses are zero-sized and unconstrained ex suppositione, their basic kinematics is of quantities of straight-line motion, or time derivatives of their rectilinear displacements. The key grounding assumptionwhich Kant in Phoronomy grasped right away, and so we need to recover as we update his 'phoronomic' foundations-is that these quantities are additive; more generally, that they are linear combinations. (His preferred term for their additivity was 'composition.' Being directed magnitudes, he thought their additivity, $\oplus$, required explanation.) In light of that, a fundamental fact is that linear velocity $\mathbf{v}_{i}$ is resolvable:

$$
\mathbf{v}_{i}=\mathbf{v}_{x} \oplus \mathbf{v}_{y} \oplus \mathbf{v}_{z} .
$$


In words: it can be decomposed into, and recovered from, its projections, viz. component velocities relative to individual rigid coordinate axes $x, y, z$. Another basic fact is that velocity of translation can be parallel-transported: ${ }^{4}$

$$
\mathbf{V}_{B \text { in } \mathrm{F}}=\mathbf{V}_{\mathbf{F} \text { relative to } \mathrm{G}} \oplus \mathbf{V}_{B \text { in } \mathrm{G}} \cdot
$$

This is really an expression for the Galilean transformation, the framing principle of classical particle kinematics. It licenses redescriptions of motion across inertial frames of reference. A last basic principle is that linear acceleration too is additive:

$$
\mathbf{a}_{t}=\mathbf{a}_{1} \oplus \mathbf{a}_{2} \oplus \ldots \oplus \mathbf{a}_{n}
$$

Namely, it is the vector sum of the single accelerations induced by individual mechanical agencies separately. In sum, the kinematic insights associated with this approach are: three degrees of freedom; and composition, or vector addition (with resolution as its correlate).

Dynamics. Kant called 'Mechanics' what we know as kinetics. Updated and tailored for the object of this approach, its 'mechanical' properties are these. The full-fledged mass-point is endowed with two action-kinds, repulsion and attraction. Both are central forces in the weak sense, i.e. they emanate from the point where the mass resides-because they are induced through charges carried by that point. Usually, these forces are the negative gradients of scalar-field potentials:

$$
\mathbf{f}=-\nabla \mathbf{P}
$$

Just as usually, these potentials are irrotational. Then the forces are central in the strong sense: they point toward or away from the center, and their vectors lie on the straight line between the mass-points that exert and undergo them, respectively. Another to describe these strong-central forces is by let-

\footnotetext{
${ }^{4}$ In the indices, 'in' denotes velocity of mass points in respect to a frame, and 'relative' denotes velocities of frames relative to one another.
} 
ting them be linear functions of some inverse power of the distance $r$ between interacting particles: ${ }^{5}$

$$
\mathbf{f} \propto c \cdot 1 / r^{\mathrm{k}}
$$

If a mass-point exerts both attraction and repulsion, and their respective force laws are species of $\mathbf{f}$ as in [5] above, it is easy to see that, on every radius from the mass-point, there is a point $P$ at some distance $b$ where the two forces balance each other: their respective accelerations cancel out. For irrotational potentials, the geometric locus of all these zero-acceleration points is a surface, viz. a 2-sphere with the mass-point at the center. This sphere is that which the young Kant in his lucid moments called the "sphere of activity" of a physical monad.

Beside laws of force-species as above, there are two dynamical laws of force generaliter. One is a version of Newton's Lex Secunda; more exactly, it is a conjunction of two statements:

$$
\mathbf{f}_{i}=\mathrm{m} \cdot \mathbf{a}_{i}
$$

In words, any force equals the mass of its target-point times the acceleration it would induce on the mass-point if it were the only force acting on it. And also,

$$
\mathbf{f}_{t}=\mathbf{f}_{1} \oplus \mathbf{f}_{2} \oplus \ldots \oplus \mathbf{f}_{n}
$$

That is, impressed forces are linear-additive magnitudes. Often and tacitly, these two are stated together as the Second Law for mass-points:

$$
\Sigma \mathbf{f}_{i}=\mathrm{m} \cdot \mathbf{a}_{t}
$$

\footnotetext{
${ }^{5}$ Below, ' $c$ ' is a placeholder for algebraic combinations of dynamical factors-broadly speaking, types of charge and/or ratios of charge to mass. Uppercase boldface letters denote tensors, viz. $3 \times 3$ matrices, lowercase boldface vectors, and lowercase italics scalars.
} 
Namely, the resultant net force on a particle equals its mass times its effective acceleration. This ultimate dynamical principle of one-way action has a complement for interactions, the Third Law:

$$
\mathbf{f}_{A B}=-\mathbf{f}_{B A} \text {. }
$$

The force-vector on a mass-point $B$ caused by a mass-point $A$ is equal and opposite to the force-vector induced by $B$ on $A$. In the standard case of masspoints with strong-central actions, the constraint [5] on force-laws entails: ${ }^{6}$

$$
\left(\mathbf{r}_{J}-\mathbf{r}_{K}\right) \times \mathbf{f}_{J K}=0
$$

I.e., the impressed force by a mass-point $J$ on another $K$ is in the straight line between them. In turn, [10] together with the principle [9] make up a version suitably called the Strong Third Law: the mutual forces on two mass-points are equal, opposite, and collinear.

Objectivity. Finally, let us consider updating Kant's Phenomenology for mass-points. Their kinematic quantities take different values depending on the frame to which we refer them, so the question arises, which of these quantities are objective? Put differently: what features of motion are observer-independent?

In our time there are two broadly overlapping approaches to objectivity for these mechanical objects. 1) Determine what quantities of motion are Galilean-invariant: roughly, those having the same value for all the functions mapping a system of mass-points from one inertial frame into another while leaving their mechanical properties intact (McKinsey \& Suppes 1955). From this standpoint, magnitude of linear acceleration counts as objective. 2) Establish the kinematic quantities inferable (from initial conditions) by means of the dynamical laws. Notoriously, these laws entail Newton's Corollary V, which means that only acceleration is measurable by all observers. There is a complication here: this route allows certain unobservable linear accelera-

\footnotetext{
${ }^{6}$ The vector $\left(\mathbf{r}_{J}-\mathbf{r}_{K}\right)$ is the distance between the two mass-points $J$ and $K$.

${ }^{7}$ I mean independent of particular observers. There is also a generic observer-the 'transcendental subject,' or 'subjectivity' in general. In Kant's doctrine, all kinematic quantities are subjectivity-dependent. But of course not all vary with particular subjects.
} 
tions. ${ }^{8}$ With that proviso in mind, it is plain that both approaches single out the same quantity. Thus, we may conclude, acceleration is objectiveperhaps up to a common linear acceleration factor-but not velocity or position.

I move now to the next framework, to see what it requires. It too has four parts, which function as modern analogues of Kant's four disciplines in Foundations, adapted to continuous matter.

\section{The deformable continuum approach}

The other paradigm of classical theory is continuum mechanics. Let us begin, again, with material constitution. To study extended bodies, continuum theory begins with an 'Euler cut,' a finite volume $\Delta V$ mentally carved out inside the body by a suitable intersection of planes or regular surfaces. There are two grounding assumptions. First, there exists a scalar function $\rho$, or mass density, defined everywhere in the cut, positive at every point; and also various charge density functions, also continuous everywhere. Second, any two material points in the cut can be moved relative to each other. ${ }^{9}$ To handle this entity mathematically, we shrink it to an infinitesimal size as a volume element, $d V$, which has a true size. In this theory macroscopic bodies are continuous wholes, and volume elements are their least, potential parts.

Kinematics. Because continuous matter is extended and deformable, its geometric behavior is much more complex than any possible motion of single mass-points. The term 'transplacement' is sometimes used for it, and it denotes a passing from a 'reference' to a 'current' configuration. A configuration is a mapping of a continuous body $B$ into a Euclidean frame $\mathbf{E}^{3}$ at some instant $t$.

To stay with Kant's preferred language of 'composition' in Phoronomy, a transplacement $\chi$ is any linear combination

$$
\chi=\mathbf{F} \oplus \mathbf{t} .
$$

\footnotetext{
${ }^{8}$ By Newton's Corollary VI, a set of interacting mass-points may be subject to system-external forces that induce (at least within the boundaries of the system) a field of (nearly) equal and parallel acceleration: e.g. gravitational attraction by a very large body at a very large distance. ${ }^{9}$ The first constitutive assumption distinguishes continuous matter radically from mass-points; the second makes it radically distinct from rigid bodies.
} 
Namely, a continuous-body motion is composed of a deformation $\mathbf{F}$ and a rigid translation $\mathbf{t}$. Either can be zero, of course. Translations occur in masspoints too, and are well understood. The topic of central interest in continuum mechanics is deformation, whose fundamental measure is the gradient $\mathbf{F}$. To see what $\mathbf{F}$ means, start with the notion of a line element, or infinitesimal fiber, $d s$. That is any material line segment whose end-points are two infinitesimally close points $P$ and $Q$ in the body. At any point $M$, the deformation gradient maps fibers (terminating in $M$ ) from the reference into the current configuration. ${ }^{10}$ Globally, $\mathbf{F}$ informs about the change of shape and orientation of a continuous body. Locally, it tells us how fibers at $M$ stretch, shrink and change mutual angles, i.e. rotate relative to each other. More exactly, let a curve $s$, parametrized by $\mathbf{z}(s)$, pass through $M$ in the reference (undeformed) configuration; and let $\mathbf{x}(s)$ be the arc-length parameterization of the same curve in the deformed configuration. The deformation gradient $\mathbf{F}$ is the tensor of the partial derivatives of the 'deformed' fiber $d \mathbf{x}$ with respect to the 'undeformed' fiber $d \mathbf{z}$ :

$$
\mathbf{F}=\partial \mathbf{x} / \partial \mathbf{z}
$$

Another useful way to gain insight into $\mathbf{F}$ is to think of $d \mathbf{z}$ as the vectors tangent to all the curves $s$ passing through $M$ in the reference configuration; and of $d \mathbf{x}$ as the same tangent vectors in the current (deformed) configuration.

A basic idea in continuum kinematics is the polar decomposition:

$$
\mathbf{F}=\mathbf{R} \cdot \mathbf{U}
$$

That is, a deformation can be seen as the product of a stretch tensor $\mathbf{U}$ and a rotation tensor $\mathbf{R}$. Stretching and shearing line elements around a point results in strain, the basic kinematic state induced by stresses and inducing

\footnotetext{
${ }^{10}$ If $\mathbf{F}$ has the same value at every point, the deformation is homogeneous, and inhomogeneous if $\mathbf{F}$ varies independently across the body.
} 
stress in return. There are several gauges of strain; in elasticity and solid mechanics, a common measure is the (right) Cauchy-Green strain tensor $\mathbf{C}:{ }^{11}$

$$
\mathbf{C}=\mathbf{F}^{T} \cdot \mathbf{F}=\mathbf{U}^{2}
$$

Thus strain can be gauged either from the deformation gradient at that point or just from the amount whereby line elements stretch or contract.

Dynamics. Mechanical continua exert two distinct kinds of actions. One is body force, a kinetic efficacy induced by a source directly on any point inside a continuous body; such is gravity or electromagnetism. Another type is contact stress, or traction. These act on the bounding surface of the body or the Euler cut, and are exerted indirectly-through the transmission of stress-on points inside the body. ${ }^{12}$ Their effect is to stretch or compress lines, change angles, bend surfaces, and alter volumes: generally, to deform.

Dynamical laws must then take into account this duality of force. There are three principles governing the kinetic actions of continua. (Five, if we include thermal phenomena, which I leave out here.) One is Conservation of Mass, a conjunction of two theses. Here is one: ${ }^{13}$

$$
m=m^{*}
$$

That is, the mass of a continuous body is invariant in all Euclidean frames. The other follows from it and the concept of deformation. It is a principle known as the Continuity Equation. I give the local form in the material description: ${ }^{14}$

$$
\rho_{0}=\mathbf{J} \rho
$$

\footnotetext{
${ }^{11} \mathbf{F}^{T}$ is the transpose of the tensor $\mathbf{F}$.

${ }^{12}$ Strictly speaking, both types are really force densities (volumetric and areal, respectively). For each kind, values at a point are obtained by taking limits from values over volumes-or areas, for tractions-around that point.

${ }^{13}$ Henceforth the asterisk denotes how an observer in rigid Euclidean motion relative to another sees or measures the same quantity as the first observer.

${ }^{14} \rho_{0}$ is mass density in the reference configuration, $\rho$ in the current (deformed) configuration, and $\mathbf{J}$ is the Jacobian of the deformation gradient tensor $\mathbf{F}$.
} 
The insight behind it is that, as mass remains constant throughout the stress action, only deformation can change mass density in a continuous body. The second fundamental principle is Cauchy's First Law, or the Balance Law of Linear Momentum. In essence it generalizes Newton's Lex Secunda in light of the dichotomy of force in continuous matter:

$$
\rho \mathbf{a}=\nabla \mathbf{T} \oplus \rho \mathbf{b}
$$

Namely, the rate of change in linear momentum of the body equals the gradient of the external stresses plus the body forces acting on it. The last basic principle is Cauchy's Second Law, or Balance of Angular Momentum. It grounds the way in which efficient causes turn continuous volumes around axes of rotation:

$$
\mathbf{r} \times \rho \mathbf{a}=\mathbf{r} \times \nabla \mathbf{T} \oplus \mathbf{r} \times \rho \mathbf{b}
$$

In words, the time-rate of change in angular momentum-around an arbitrary point at distance $\mathbf{r}$ from the body-equals the net torque of the body forces and contact stresses on a continuous body.

Objectivity. As the kinematic possibilities of continua far outnumber those of mass-points, the question of mechanical objectivity-the topic of an updated Kantian Phenomenology-for a continuous system poses new challenges. It really has two aspects. One regards the objective behavior of a special 'representative' point in the body: its mass center, or any centroid more generally. This aspect considers the body as a particle, so the question has the same answer as it did for mass points-linear acceleration is objective, position and velocity are not. But this easy victory wins little; by reducing the body to the motion of its centroid, we leave unexplained most of material behavior, and turn extension and continuity into idle notions.

To avoid that, we must include true shape and stress. A different concept of objectivity arises then. To grasp it, start with the notion of change of Euclidean frame: let $O$ and $O^{*}$ be observers respectively at rest in two rigid frames in arbitrary relative motion. Let the following be functions of time: a point function $\chi$, a symmetric tensor $\mathbf{T}$, a vector $\mathbf{c}$, and a proper orthogonal 
tensor $\mathbf{Q} \cdot{ }^{15}$ Let $O$ observe $\chi$ and $\mathbf{T}$ at some time $t$. Then $O *$ will see these two magnitudes as $\chi^{*}$ and $\mathbf{T}^{*}$ given by:

$$
\begin{aligned}
& \chi^{*}=\mathbf{c} \oplus \mathbf{Q} \cdot \chi \\
& \mathbf{T}^{*}=\mathbf{Q} \cdot \mathbf{T} \cdot \mathbf{Q}^{\mathbf{T}}
\end{aligned}
$$

Intuitively, this says that $O$ and $O^{*}$ are in rigid motion relative to each other as they observe the same two functions. ${ }^{16}$ Next, define a dynamical process $D$ of a continuous body as a pair of fields $[\chi, \mathbf{T}]$ for a given assignment $\rho$ of mass density; and let $D^{*}$ be a process $\left[\chi^{*}, \mathbf{T}^{*}\right]$ for the same $\rho$. If their respective fields are related as in [18a-b] above, the two processes are said to be equivalent. To say that $\boldsymbol{D}$ and $\boldsymbol{D}^{*}$ are equivalent means that the same physical process is described by two different arbitrary observers.

To complete the account, we need the idea of constitutive relations. Intuitively, they are differential equation-relating stress and strain-that describe possible basic ways in which continuous matter responds to applied stresses. An infinite number of such ways are possible mathematically, soto delimit the class of physically admissible relations-an objectivity condition is imposed. This constraint is called 'material frame-indifference.' One way to state it is, "constitutive relations must be invariant under changes of reference frame" (Malvern 1969: 389). Namely, if a dynamical process $D$ satisfies a constitutive relation $F$, then $F$ must also be satisfied for any other equivalent process $D^{*}$ as defined above. The crucial thing to note then is that a mechanical theory of continuous matter needs two concepts of objectivity, or observer-independence: Galilean and Euclidean.

\section{Via regia, and the road not taken}

It should be clear by now that the two approaches I outlined above are mutually irreducible, since their respective basic objects are radically distinct. One is discrete, zero-sized, exerting only Galilean-invariant actions at a distance.

\footnotetext{
${ }^{15}$ A tensor $\mathbf{Q}$ is properly orthogonal if the product $\mathbf{Q} \cdot \mathbf{Q}^{T}$ with its transpose equals 1.

${ }^{16}$ Because $\mathbf{c}$ is a rigid translation and $\mathbf{Q}$ a rigid rotation or mirror reflection. In other words, [18a-

b] are the Euclidean transformations.
} 
The other is continuous, extended, and capable of Euclidean-invariant contact actions. Kant's foundations cannot accommodate both objects. The reason is his grounding approach: the categories must be applied to matteranalyzed as mobile, impenetrable, momentum-carrying, observerindependent objecthood-so as to yield determinate univocal spatiotemporal content, viz. geometric magnitudes and their time derivatives. We do so by specifying the geometric makeup and kinematic content of the terms 'body,' 'motion,' 'force,' and 'action.' But, a univocal specification yields a uniquely defined content, and so matter will be either mass-points or deformable continua, not both. So, we must decide which of these two is more firmly anchorable in his metaphysics.

One might object that my question has a foregone conclusion: Kant chose continuous matter explicitly. In Foundations, he claims to prove that mass distribution is continuous at all scales: "Matter is divisible to infinity, and in fact into parts such that each is matter in turn" (4:503). And, he argues strongly against "physical monads," i.e. mass-points. So, it is clear which path to neoclassical mechanics is legitimate from his standpoint. Still, this is far from conclusive. For one, Kant does not much examine whether his preferred theory of matter coheres with the rest of his foundations, and the truth is that it does not (Stan 2014). In fact, a close look at his demarche in Foundations reveals that Kant vacillates between the two approaches I have outlined, though he seems unaware of that. Thus, my question stands. Then let us examine both approaches, and assess their respective chances and interpretive costs.

Mass-points. Grounding their kinematics is uncommonly easy, for Kant has done so already, though unintentionally. In 'Metaphysical Foundations of Phoronomy,' he starts with mobility as an essential property of body, and goes on to justify the key principle for the 'composition of motions,' viz. an addition rule for linear velocity, which he regards as the basic quantity. But, he legitimizes that conception with a sleight of hand:

Since in phoronomy nothing is to be at issue except motion, no other property is here ascribed to the subject of motion, namely, matter, aside from movability. It can itself so far, therefore, also be considered as a point, and one abstracts in phoronomy from all inner constitution.... If the expression "body" should nevertheless sometimes be used here, this is only to anticipate to some extent the ap- 
plication of the principles of phoronomy to the more determinate concepts of matter that are still to follow. (4:480; my italics)

Satis non est. Point motion is just change in three degrees of kinematic freedom. Kant's doctrine is that bodies are continua, but those are inherently extended, and have shapes irreducible to points. Ergo, Phoronomy properly conceived will be a theory of change in shape. At the very least, Kant ought to have made it a theory of infinitesimal fibers $d s$ and their relative motions. Then the basic concept of Phoronomy becomes deformation, not point motion as he claims. His claim above does not follow from his argument, but is licensed by two other considerations. (A) In some contexts, we may abstract from the body's rotations and deformations and treat it as a mass-point, by letting the point-motion of a centroid stand for the motion of the extended body itself. That is precisely Kant's practice in Foundations, where his preferred centroid is the mass center-because he aimed to ground Newton's approach, who in Principia replaces any extended planet with an (extensionless) mass-point. (B) Or we may regard the body as composed of masspoints, and then its motion is the sum of its constituents' point-motions.

Be that as it may, the fundamental kinematics for the approach (A) is very much the basis for (B) too, and so Kant's mistaken conclusion above becomes his felix culpa. It led him to a composition theorem equivalent to [1] above. To see that, let a test particle move simultaneously relative to three frames themselves in mutual uniform translation at right angles. The particle's three motions-relative to each frame-can now be treated as its component velocities, and so its 'composite motion,' as Kant would call it, becomes its linear velocity $\mathbf{v}$ as in [1], relative to a (fourth) frame supposed stationary. Thus in the course of explaining how motion becomes a magnitude, Kant justified the fundamental result in particle motion. Even better, if we regard the motions in Kant's account as velocities acquired from rest by a particle, it turns out that his explication of 'composite motion' can also justify the equality [3] above. And, because Kant took 'composite motion' to be at bottom an addition of inter-frame velocities, naturally his notion also explains the Galilean transformation [2] above. Consequently, Kant has already grounded the basic kinematics for mass-points, and the interpreter need only re-embed it in a broader explanatory framework. 
The dynamics stage of my inquiry must follow two tracks. We may wonder first about the chances of embedding in his doctrine the two force species-themselves generic-proper to mass-points. Here too the official Kant is already of great help. A constant of his natural philosophy was that 'force' is constitutive of matter. ${ }^{17}$ One such agency, 'attraction,' is just the kind of force that mass-points exert too: central, induced by a potential, acting at a distance. Kant conceived it on the model of gravity, but was non-committal enough to leave its specifics open. Thus he would agree that the law of his 'attraction' takes the general form as in [5] above. However, his other dynamical agency, 'repulsion,' defies the interpreter, for in the 1780 s he made it a contact force, thus incompatible with the mass-point approach. I have two remarks to help overcome this obstacle. First, Kant's account of repulsion is not self-standing. It is a corollary of his broader view that matter is a physical continuum: "divisible to infinity... into parts such that each is matter in turn," he argues in Proposition 4 of Dynamics (4:503). But, his master argument for continuous matter in that Proposition is not unassailable; if we examine it critically, it may turn out to be flawed. ${ }^{18}$ Second, even if his argument is good, we can discount it as part of the price for extending his foundations to make room for modern mechanics. It is a small price, really: keep his force of repulsion, discard his continuity-of-mass thesis, and the result is repulsive force fit for mass-points: just like his attraction, it would be central, acting at a distance. Then its force-law will be a species of [5] above. So, it can balance Kant's fundamental 'attraction,' thereby co-grounding a notion of least effective volume as mass-points have. After all, mass-points used to be Kant's theory of matter in Physical Monadology. ${ }^{19}$ And so, dropping Proposition 4 gets the interpreter a ready-made basis for half of basic particle mechanics.

What about the other half, the general laws of impressed force? Here too the landscape is friendly. Kant in his Phoronomy gives grounding for [3], viz. the additivity of linear acceleration. He offers that as the "a priori basis" for another key result, the Parallelogram of Forces. Though he does not carry

\footnotetext{
${ }^{17} \mathrm{He}$ meant that 'force' is a basic attribute - with mobility-in that bodily extension, impenetrability, and ability to transfer momentum are explainable from 'force.'

${ }^{18}$ For instance, Friedman - virtually alone in having examined it-seems to me hesitant to declare it sound (2013: 150f.). I take comfort in that.

${ }^{19}$ Or one of them-apparently, he flirted with two theories in that paper; cf. Smith 2013.
} 
out an actual derivation-alluding instead to the collinearity of force and its induced acceleration-Kant is sure that it can be done, and so are some interpreters. ${ }^{20}$ If so, Kant has thus secured [7], the additivity of impressed force. Still, that is not yet enough for the Second Law. We must still identify Kantian resources for [6], the Lex Secunda for single forces. Perhaps an argument can be made-broadly in tune with the Kant of Friedman's recent work - that [6] is not really a law but an axiom: a postulate, or physical primitive, posited for the sake of rigorous experience. Or, as Friedman has it, for making possible the application of mathematics to nature, by exhibiting how force becomes a magnitude, i.e. acquires a ratio structure (2013: 237). So, we have prima facie reasons to be hopeful about extending to mass-points this part of Kant's foundations, though of course it needs more work. ${ }^{21}$

Discarding his Proposition 4 as I urged above would also make the Strong Third Law at home in Kant's metaphysics of nature. He already has a good argument for [9], the weak version of Lex Tertia. With attraction and repulsion reconceived as actiones in distans, it is easy to show these Kantian basic powers are strongly-central. We need only rely on an idea already available to him, specifically Lagrange's $\Omega$-function (Lagrange 1777). The function is a mathematical description of the basic agency exerted by masspoints: a scalar potential whose gradient is a conservative, irrotational vector field. In physical terms, if attraction and repulsion are $\Omega$-functions, they obey [4]; just re-label ' $P$ ' as ' $\Omega$ ' to see that. But [4] and [9] entail [10], which means that Kant's mature metaphysics-with physical monads, the direct consequence of deleting Proposition 4-support the Strong Third Law, hence the two basic laws of point-mass dynamics.

And so we come to objectivity and the task of extending Kant's Phenomenology. The prolegomenon to a solution, I believe, is to note a deep ambiguity in his theory of motion. On the one hand, modern interpretations (based on Friedman's highly consilient reading) strongly imply that Kant lets true mo-

\footnotetext{
${ }^{20}$ See Friedman (2013: 377-9) and Stan (2014: 429). Still, we should give this matter careful scrutiny. Attempts to ground the Parallelogram of Forces are notoriously thorny, and it is not yet clear that, even by 18th-century standards of physical proof, Kant's derivation would succeed.

${ }^{21}$ Additionally, we would have to supplement Kant's conception of mass. In the 1780s, he thinks of it just in terms of active powers - of transferring momentum and 'imparting motion' by acceleration fields-but hardly as the measure of resistance to acceleration. That is unfortunate especially as in Physical Monadology Kant had a clear conception of mass as resistance to force.
} 
tion be the kinematic quantity satisfying his 'mechanical laws.' This idea coupled now with a ontology of mass-points entails that true motion is that which arbitrary inertial observers would measure by applying the laws [6] through [10]. Specifically, it is linear acceleration, because those laws are Galileanrelative. On the other hand, Kant throughout his philosophical maturity was wedded to the early modern idea that the laws of motion are 'true relative to' a preferred frame, viz. absolute space. However, this makes true motionthus objective behavior-be a preferred velocity, or rate of change in distance to the privileged frame. That runs against the first strand of thought above, and also against our modern view of objectivity.

That is the conundrum. To break it, I recommend adopting the modern view that Kant lets the laws themselves define objective mechanical behavior; and adjust the Phenomenology—construed à la manière de Friedmanto the dynamical laws of point masses. As far as I can tell in advance, the only drastic correction will involve Kant's doctrine of necessary motion, admittedly the acme of Phenomenology. Officially, Kant construes it as true velocity, whose necessity is allegedly conceptual: it "follows immediately and unavoidably from the concept of the relation of the moved in space to anything else movable thereby" (4:558, my italics). ${ }^{22}$ That becomes untenable in the project pursued here. Like Friedman, we ought to re-read 'necessary motion' as true acceleration, whose necessity is special-metaphysical, or grounded in Kant's 'special metaphysics of material nature.' The Second Law, now added explicitly to that edifice, entails that any force on a body, or set of masspoints, induces an objective acceleration on it. Lex Tertia then makes it necessary that any body experiences an objective acceleration in proportion to its action on other bodies, because all impressed forces necessarily are interactions, by the Third Analogy. ${ }^{23}$

Continuous matter. Let us now move on to the other paradigm, and assess it for ease of retrofit to Kant's metaphysical foundations. It seems we should be hopeful about grounding its fundamental kinematics. Recall that a

\footnotetext{
${ }^{22} \mathrm{He}$ means that, just by a conceptual analysis of 'relative motion,' we can establish that two interacting bodies have true velocities relative to each other, in the mass-center frame (Stan 2013).

${ }^{23}$ It is not clear what role is left for Kant's absolute space to play. The dynamical laws pick out a family of equivalent frames, not a single preferred reference system.
} 
continuum motion generaliter is composed of a rigid translation $\mathbf{t}$, a rigid rotation $\mathbf{R}$ and a pure stretch $\mathbf{U}$. And, Kant sees his task in Phoronomy precisely as supplying a priori principles to exhibit the "concept of a composite motion" in pure intuition (4:487). So, we might expect the core grounding to be in place already. But this hope is short-lived. Kant sees composition of motions as homogeneous in two senses: it is a uniform operation, vector addition; and is a "composition of the homogeneous" in that it adds up motions of the same kind (4:495; cf. also Sutherland 2014). But continuum motion is heterogeneous on both counts. It involves vector addition and tensor multiplication; see [11] and [13] above. And, it combines different species of displacements. More seriously, Kant's basic kinematic object is of the wrong kind for continua. His relevant entity is "the motion of a point" (4:489). But in continuum kinematics the fundamental object is the infinitesimal fiber, not the point, so any interpretive attempt to anchor continuum motion in his metaphysical foundations of phoronomy is bound to violate the letter of Kant's doctrine. Perhaps we can retain its spirit, at least? For instance, by discarding wholesale Kant's outdated geometric approach to kinematics, and replacing it with 'composition' as in linear algebra, i.e. as combination of elements in a vector space. I cannot judge the feasibility of this proposal in advance, but I must warn that ultimately it will need anchoring in Kant's categories of quantity, as his foundational program requires. Thus, so far continua seem a good deal harder to embed in his phoronomy.

Next, it appears that updating his Dynamics, or theory of matter, is easiest. After all, he does have the continuity of mass and a distinction between 'penetrating' and 'surface' actions, i.e. body forces and stresses (4:516). And yet, even here some reconceptualization is needed. Kant really ought to start with a notion of force density - specifically, amount of body force per unit volume and of stress per unit area. Values at a point, needed for equations of motion, are then obtained by taking limits, viz. letting these units shrink to a volume element $d V$ and area element $d A$, respectively. Thus, not even this chapter of Foundations would survive intact the evolution of subsequent mechanics.

On to the dynamical laws, then. Kant saw insightfully that a mechanics of continua needs Conservation of Mass, which he linked firmly to his meta- 
physics through the First Analogy of Experience. But, his architectonic approach to grounding yields gains and losses. If we follow his lead, we can at best generalize his procedure to cover inertia of rotation and the equality of impressed torques. However, it is far from clear how the interpreter might make room for principles that really drive theory-building in this mechanics, viz. Cauchy's two laws [16] and [17] above. The latter principle is probably not out of bounds for Kant: it is derivable as a theorem, provided we allow on Kant's behalf that the stress tensor T, the basic measure of contact action, is symmetric. Still, that raises again, more pressingly than ever, the question whether the Second Law for single forces is anywhere in Kant's metaphysical foundations, and how in its absence he might be credited with it.

I think there is a heuristic out of this predicament, and it appears feasible. Friedman once ascribed to Kant the view that Lex Secunda was implicit in his 'mechanical' laws of inertia and of action-reaction (1992: 143). I suggest reviving that idea in the context of rational reconstruction rather than textual hermeneutics. ${ }^{24}$ More generally, I think we may regard the Second Law (and its correlate for rotation) as "bridge laws" between the purely metaphysical, qualitative principles of force and action, and the mathematically-redescribed 'mechanical' appearances, i.e. phenomena of motion conceived as transplacements. This would keep the spirit while correcting the letter of Kant's Foundations as an explanatory project of how mathematics can apply to appearance and turn it into mechanical experience, paradigmatically.

Still, I am hard pressed to see any Kantian path to grounding objectivity for continua. Granted, Kant apparently had a relativity principle compatible with Newton's Corollary Six. However, even that is too weak for the objectivity at issue here, which is really Euclidean relativity, as it were: invariance across rigid frames, translating and rotating relate to each other. But Corollary Six holds only in a proper subset of the equivalence class of all Euclidean frames. Nor is there any hint of how this notion might be reconciled with Kant's categories of modality. Because continuum objectivity does not single out inertial frames, the interpreter cannot just transfer the stepwise approx-

\footnotetext{
${ }^{24}$ There is textual support for my suggestion. Kant regards his 'dynamical' repulsion and attraction as forces of "imparting motion" whereby they generate momentum increments. And, his indirect proof of the Equality of Action and Reaction assumes that actions are impressed forces that generate linear accelerations (4:536; 562f.). These claims presuppose the Second Law.
} 
imating procedure outlined in the Phenomenology—as Friedman and others read it - and invoke the modal category appropriate for each stage.$^{25}$ Continuum objectivity is a constraint meant to determine 'real' or 'intrinsic' material properties-codified by the body's response to stress, not just by the motion of its mass center, which Kant singled out for treatment in Phenomenology. It is the presence and special nature of stress and strain, which masspoints lack and cannot support, that requires a second, distinct concept of objectivity for continuous matter.

\section{Balance and conclusions}

In sum, to ground continuum theory in Kant's metaphysics of nature, we would need to discard his Phoronomy and replace it wholesale; retool the mathematics of his Dynamics - and generally replace his geometric methods with the partial-differential calculus; supplement his Mechanics with two highly non-trivial principles; and regard his Phenomenology as incomplete, in need of extension by another concept of objectivity, material frameindifference.

In turn, adopting mass-points would leave the Phoronomy mostly unaltered; leave the Dynamics in place, save for the untenable Definition 4; require us to make explicit the source and status of the Second Law in his Mechanics; and prompt us to clarify his idea of true motion in Phenomenology.

With the initial estimate of interpretive costs behind us, which of the two approaches is preferable? On balance, mass-points appear a good deal less radical and invasive. Continuum theory requires strong foundations, and I am really not sure that Kant's metaphysics can provide them or even that it needs to do so.

Another point against the continuum approach is that Kant's project pursues a stringent notion of metaphysical grounding, and so it places strong constraints on any attempt to extend or update it. Specifically, explanation in it flows from the categories and transcendental principles, through the concept of matter and its attributes, to basic mechanical theory. But, keeping continuous matter makes it very hard to live up to this ideal, hence give a

${ }^{25}$ Of course, inertial frames remain indispensable - the laws of continuum mechanics hold just relative to them, not generally. 
Kantian grounding for neoclassical mechanics. That is because, if we keep Kant's physical continuum, we must supply what he lacks-everything else, really. In effect, we would have to build fundamental kinematics and dynamics, the results [11] through [18] above, from the ground up, i.e. from Kant's analysis of matter-and then retrofit them to his constitutive apparatus imported from the First Critique. How to carry this out is utterly unclear to me, yet it must be done or else we betray Kant's foundational thrust, which renders hollow any victory so obtained. In contrast, mass-points satisfy Kant's strong constraint very well. He had already shown-accidentally, as he rejected them in theory-that they instantiate his categories of quantity, relation, and modality. It is quite easy now to argue that they cohere with the categories of quality too: they bear the same basic forces as his continuum, and the continuity of mass distribution has no import on category-instantiation.

Here is another reason to extend Kant in line with the first approach. Note how little disruptive it is to anchor mass-point mechanics in his doctrine. That is because the doctrine is really a palimpsest. The architectonic of categories gives it a monolithic façade, but behind it two different modes of thought, particulate and continuous, are superimposed. Thanks to Smith (2013), now we know that Kant always wavered between these two views of matter. Officially a mass-point theorist in Physical Monadology, Kant there at times lets the continuum picture take over unannounced. In Foundations, he commits to continuous matter, and yet he cannot let go of the particulate approach specific to mass-points. Then, seeing as his foundation is really a heterogeneous blend, the interpreter should not be afraid to purify it by removing extraneous ingredients. In my view, taking the mass-point approach amounts to proper purification: it retains the essence of Kant's philosophy of mechanics while increasing its strength. In contrast, the continuum approach would dilute it beyond recognition merely to preserve just one commitment out of so many that Kant expressed in Foundations.

A last but compelling reason to embed mass-points into Kant's metaphysics is that they make continuum theory foundationally dispensable. This is not immediately apparent, but emerges in hindsight from the long-term growth of mechanics. Modeling matter as continuous is needed just at certain scales, not universally, and mass-points have proven resilient enough to 
allow that. Specifically, modern mathematical tools, calibrated for the desired scale, enable mechanics to start with discrete point masses and define from them continuous fields as needed. ${ }^{26}$ So, it turns out that mass-points are the better matter theory for classical dynamics, and the physical continuum is a local, reducible phenomenon. Then we need not saddle Kant with it as we bring him into the present.

I end with a parting note, not unimportant. Readers will wonder how my key thesis - that we ought to commit Kant to a kind of physical monads-is compatible with his Second Antinomy and its injunction against them. My answer is brief here, but it deserves elaboration elsewhere. In two respects, my result is orthogonal to Kant's concerns in the Antinomy. For one, I do not advance it as a thesis in transcendental realism, viz. about the architecture of matter as Ding an sich. On the contrary: it is about the microstructure of the substratum, itself phenomenal, of all appearances in outer sense. It recommends an ontology inferable from phenomena - of motions and deformations given at mesoscopic scales and above-by a systematic use of Kant's Postulates of Empirical Thought. Then my result is in line with his broader project in the Analytic, just as I promised at the outset. Because of that (now this is another respect) my reasoning is safe from Kant's censure in the Antinomies. I did not reach my conclusions by mere analysis from mere concepts, aided by the Principle of Sufficient Reason-as Wolff and the practitioners of 'rational cosmology' did, thus earning Kant's rebuke. Rather, I ascribed to Kant mass-points as the best ontological explanation of all determinate phenomena of motion in a Galilean regime. As such, my inference is beyond Kantian reproach, for it does not claim for itself any greater certainty than he believed he had secured for his own principles in Foundations.

In conclusion, there is a promising case that Kant's 'special metaphysics of material nature' remains viable and relevant. As I have explained, that is due to his insightful grounding of mechanics in a robust, resilient constitution theory of mechanical objecthood; and to the changing but ultimately happy fortunes of classical mechanics itself. In regard to the former, Kant's farsighted kinematic and dynamical foundations permit an ontology of mass-

\footnotetext{
${ }^{26}$ The first effort along these lines was Irving and Kirkwood 1950; the latest, most detailed expression is Murdoch 2012.
} 
points to be embedded without much disruption - though not the continua he embraced in the Critical decade. As to the latter, while Einstein did curtail the once imperial ambitions of mechanics, in the end a vast swathe of determinate experience still unfolds at speeds and scales for which classical theory remains indispensable. Thus, thanks to his relentless and deep engagement with it, Kant's mechanical foundations remain the most important legacy of early modern natural philosophy.

Acknowledgments. For helpful suggestions and enlightening discussions, I am indebted to Michela Massimi, Angela Breitenbach, Katherine Brading and Michael Friedman.

\section{References:}

Friedman, M. 1992. Kant and the Exact Sciences. Harvard University Press.

Friedman, M. 2013. Kant's Construction of Nature. Cambridge University Press.

Gurtin, M., Fried, E., Anand, L. 2010. The Mechanics and Thermodynamics of Continua. Cambridge University Press.

Hamel, G. 1909. Über die Grundlagen der Mechanik. Mathematische Annalen 66: $350-97$.

Irving, J.H., Kirkwood, J.G. 1950. The statistical mechanical theory of transport processes IV. The equations of hydrodynamics. The Journal of Chemical Physics 18: 817-829.

Joos, G. 1934. Theoretical Physics, tr. I.M. Freeman. New York: Hafner.

Kant, I. 2004. Metaphysical Foundations of Natural Science, ed. and trans. M. Friedman. Cambridge University Press.

Lagrange, J.L. 1777. Remarques générales sur le mouvement des plusieurs corps qui s'attirent mutuellement en raison inverse des carrés des distances. Nouveaux Mémoires de l'Académie royale des sciences et belles-lettres, 155-72. Berlin.

Malvern, L. 1969. Introduction to the Mechanics of a Continuous Medium. Englewood Cliffs: Prentice-Hall.

McKinsey, J.C.C., Suppes, P. 1955. On the notion of invariance in classical mechanics. British Journal for the Philosophy of Science 5: 290-302.

Murdoch, A.I. 2012. Physical Foundations of Continuum Mechanics. Cambridge University Press.

Smith, S. 2013. Kant's picture of monads in the Physical Monadology. Studies in History and Philosophy of Science 44: 102-111. 
Stan, M. 2014. Unity for Kant's Natural Philosophy. Philosophy of Science 81: 423443.

Sutherland, D. 2014. Kant on the construction and composition of motion in the Phoronomy. Canadian Journal of Philosophy 44: 686-718.

Truesdell, C. 1991. A First Course in Rational Continuum Mechanics, 2nd ed. Boston: Academic Press.

Wilson, M. 2013. What is "classical mechanics" anyway? The Oxford Handbook of Philosophy of Physics, ed. R. Batterman, 43-106. Oxford University Press. 